Qingguo Meng, Chen Yue, Jiangkun Yu, Qingyun Liu and Jitao Lu*

\title{
Crystal structure of (dimethylformamide- $\mathrm{K} O$ )(perchlorato- $\left.\mathrm{K}^{2} O, 0^{\prime}\right)\left\{\mu_{2}-6,6^{\prime}\right.$ - ((1,2-phenylenebis(azanylylidene))bis(methanylylidene))bis(4-bromo-2- methoxyphenolate)- $\mathrm{K}^{8} \mathrm{~N}, \mathrm{~N}^{\prime}, 0: 0, \mathrm{O}^{\prime}: \mathrm{O}^{\prime}, \mathrm{O}^{\prime \prime}, \mathrm{O}^{\prime \prime \prime}$ \}sodium(I)nickel(II), $\mathrm{C}_{25} \mathrm{H}_{23} \mathrm{Br}_{2} \mathrm{ClN}_{3} \mathrm{NaNiO}_{9}$
}

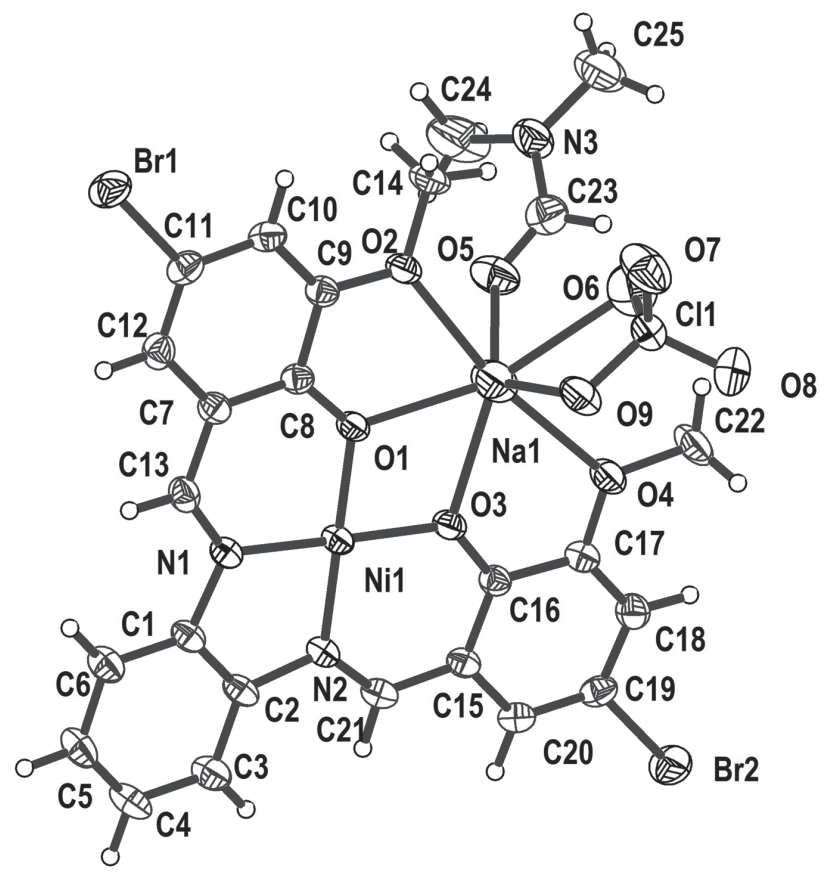

https://doi.org/10.1515/ncrs-2017-0309

Received December 2, 2017; accepted February 24, 2018; available online March 10, 2018

\section{Abstract \\ $\mathrm{C}_{25} \mathrm{H}_{23} \mathrm{Br}_{2} \mathrm{ClN}_{3} \mathrm{NaNiO}_{9}$, monoclinic, $P 2_{1} / n$ (no. 14), $a=15.595(4) \AA, \quad b=10.993(3) \AA, \quad c=18.660(5) \AA$, $\beta=114.536(4), \quad V=2910.2(14) \AA^{3}, \quad Z=4, \quad R_{\mathrm{gt}}(F)=0.0390$, $w R_{\text {ref }}\left(F^{2}\right)=0.0998, T=293 \mathrm{~K}$.}

CCDC no.: 1825595

The crystal structure is shown in the figure. Tables 1 and 2 contain details on crystal structure and measurement conditions

*Corresponding author: Jitao Lu, College of Chemical Engineering and Environmental Chemistry, Weifang University, Weifang 261061, Shandong, China, e-mail: lujitao@foxmail.com

Qingguo Meng, Chen Yue and Jiangkun Yu: College of Chemical Engineering and Environmental Chemistry, Weifang University, Weifang 261061, Shandong, China

Qingyun Liu: College of Chemical and Environmental Engineering, University of Science and Technology, Qingdao 266510, Shandong, China
Table 1: Data collection and handling.

\begin{tabular}{ll}
\hline Crystal: & Colorless block \\
Size: & $0.21 \times 0.15 \times 0.11 \mathrm{~mm}$ \\
Wavelength: & Mo $K \alpha$ radiation $(0.71073 \AA$ $)$ \\
$\mu:$ & $3.58 \mathrm{~mm}^{-1}$ \\
Diffractometer, scan mode: & Bruker SMART, $\varphi$ and $\omega$-scans \\
$\theta_{\text {max }}$, completeness: & $27.7^{\circ},>99 \%$ \\
$N(h k l)_{\text {measured }}, N\left(h k l l_{\text {unique }}, R_{\text {int }}:\right.$ & $16821,6678,0.035$ \\
Criterion for $I_{\text {obs }}, N(h k)_{\text {gt }}:$ & $I_{\text {obs }}>2 \sigma\left(I_{\text {obs }}\right), 3992$ \\
$N(\text { param })_{\text {refined }}:$ & 393 \\
Programs: & Bruker programs [1], SHELX [2], \\
& OLEX2 [3] \\
\hline
\end{tabular}

and a list of the atoms including atomic coordinates and displacement parameters.

\section{Source of materials}

The Schiff-base ligand was synthesized by condensation of 1,2-diaminobenzene and 5-bromo-3-methoxyl-2hydroxybenzaldehyde with the molar ratio 1:2 in ethanol. The synthesis of the title complex was carried out by reacting $\mathrm{Ni}\left(\mathrm{ClO}_{4}\right)_{2} \cdot 6 \mathrm{H}_{2} \mathrm{O}, \mathrm{CH}_{3} \mathrm{COONa}$ and the schiff-base ligand (1:2:1, molar ratio) in DMF. Stirring was continued for half an hour at room temperature. The mixture was filtered and the filtrate was evaporated in air without disturbed. After 1 month, block red-brown crystals suitable were obtained with a yield about $55 \%$.

\section{Experimental details}

A terminal methyl group was refined with disorder model and SADI and RIGU restrains to get better models. $U_{\text {iso }}$ values of hydrogen atoms were set to $1.2 U_{\text {eq }}$ or $1.5 U_{\text {eq }}$ of the parent atoms.

\section{Comment}

Because not only of their interesting structures, but also more important of the great potential applications in many fields, the Schiff-base ligand based complexes have all along attracted much attention [4-6]. Among which, the complexes containing paramagnetic transition ion(s) based-on the salen types of quasi-planar chelate ligands have been widely studied in the molecular magnetism field owing to 
Table 2: Fractional atomic coordinates and isotropic or equivalent isotropic displacement parameters $\left(\AA^{2}\right)$.

\begin{tabular}{|c|c|c|c|c|}
\hline Atom & $x$ & $y$ & $z$ & $U_{\text {iso }} * / U_{\text {eq }}$ \\
\hline $\mathrm{Ni1}$ & $0.02437(3)$ & $0.22736(4)$ & $0.99954(2)$ & $0.03812(12)$ \\
\hline Va1 & $0.10600(10)$ & $0.26663(12)$ & $0.85809(8)$ & $0.0530(4)$ \\
\hline $3 r 1$ & $-0.26624(3)$ & $0.74480(4)$ & $0.78779(2)$ & $0.06748(15)$ \\
\hline $\mathrm{r} 2$ & $0.35619(3)$ & $-0.28026(4)$ & $1.09436(3)$ & $0.07031(15)$ \\
\hline 11 & $0.26998(7)$ & $0.43203(9)$ & $0.85196(6)$ & $0.0574(3)$ \\
\hline 01 & $0.00350(16)$ & $0.3294(2)$ & $0.91574(12)$ & $0.0438(5)$ \\
\hline 02 & $-0.00289(17)$ & $0.4422(2)$ & 7(13) & $30(6)$ \\
\hline 03 & $0.11197(16)$ & $0.1584(2)$ & $0.96945(12)$ & $0.0458(6)$ \\
\hline 04 & $0.23071(18)$ & $0.1020(2)$ & $0.91047(14)$ & $0.0569(7)$ \\
\hline 05 & $0.0323(2)$ & $0.1807(3)$ & $0.73615(16)$ & $0.0753(8)$ \\
\hline 06 & $0.2134(2)$ & $0.3506(3)$ & $0.79185(18)$ & $0.0982(11)$ \\
\hline 07 & $0.2606(3)$ & $0.5497(3)$ & $0.8201(2)$ & $0.0994(11)$ \\
\hline 08 & $0.3643(2)$ & $0.3931(4)$ & $0.88111(19)$ & $0.1063(12)$ \\
\hline 09 & $0.2350(2)$ & $0.4291(3)$ & $0.91208(15)$ & $0.0778(9)$ \\
\hline V1 & $-0.06207(19)$ & $0.3021(2)$ & $1.02925(15)$ & $0.0389(6)$ \\
\hline N2 & $0.04339(19)$ & $0.1175(2)$ & $1.08009(14)$ & $0.0399(6)$ \\
\hline N3 & $0.0000(2)$ & $0.1853(3)$ & $0.60707(19)$ & $0.0711(10)$ \\
\hline 1 & $-0.0691(2)$ & $0.2457(3)$ & $1.09520(19)$ & $03(8)$ \\
\hline $\mathrm{C} 2$ & $-0.0118(2)$ & $0.1446(3)$ & $1.12281(18)$ & $0.0439(8)$ \\
\hline$C 3$ & $-0.0127(3)$ & $0.0792(3)$ & $1.1860(2)$ & $0.0558(10)$ \\
\hline H3 & 0.025065 & 0.010644 & 1.204327 & $0.067^{\star}$ \\
\hline $\mathrm{C} 4$ & $-0.0696(3)$ & 54(4) & 1(2) & $0.0616(11)$ \\
\hline $\mathrm{H} 4$ & -0.070255 & 0.073049 & 1.263671 & $0.074^{*}$ \\
\hline C5 & $-0.1255(3)$ & $0(4)$ & 1.1 & $588(10)$ \\
\hline H5 & -0.163191 & 0.241538 & 1.219215 & $0.071^{*}$ \\
\hline C6 & $-0.1274(3)$ & $0.2826(3)$ & $6(2)$ & 0513(9) \\
\hline $\mathrm{H} 6$ & -0.166577 & 885 & 953 & $0.062^{\star}$ \\
\hline C7 & $-0.1143(2)$ & $0.4569(3)$ & 0.9280 & 105(8) \\
\hline$C 8$ & $-0.0560(2)$ & $0.4193(3)$ & 0.891 & $0.0387(8)$ \\
\hline C9 & $-0.0624(2)$ & $0.4836(3)$ & $0.82363(18)$ & $0.0401(8)$ \\
\hline 10 & $-0.1235(2)$ & $0.5788(3)$ & $0.79397(19)$ & $0.0465(9)$ \\
\hline 10 & -0.126927 & 074 & 0.749331 & $0.056^{*}$ \\
\hline$C 11$ & $-0.1806(2)$ & $0.6133(3)$ & $0.8317(2)$ & $0.0482(9)$ \\
\hline 12 & $-0.1767(2)$ & (3) & $0.8973(2)$ & $0.0476(9)$ \\
\hline $\mathrm{H} 12$ & -0.214622 & 0.581871 & 0.921918 & $0.057^{*}$ \\
\hline C13 & $-0.1128(2)$ & $0.3965(3)$ & $0.99617(19)$ & $0.0446(8)$ \\
\hline H13 & -0.151455 & 0.427435 & 1.018706 & $0.053^{*}$ \\
\hline C14 & $-0.0123(3)$ & $0.4925(4)$ & $0.7190(2)$ & $0.0643(11)$ \\
\hline $\mathrm{H} 14 \mathrm{~A}$ & -0.074827 & 0.477303 & 0.679757 & $0.096^{*}$ \\
\hline H14B & 0.032826 & 0.455333 & 0.703260 & $0.096^{*}$ \\
\hline $\mathrm{H} 14 \mathrm{C}$ & -0.001363 & 0.578573 & 0.724724 & $0.096^{*}$ \\
\hline C15 & $0.1602(2)$ & $-0.0068(3)$ & $1.06129(18)$ & $.0394(8)$ \\
\hline C16 & $0.1648(2)$ & $0.0645(3)$ & $1.00023(18)$ & $0.0384(7)$ \\
\hline C17 & $0.2304(2)$ & $0.0283(3)$ & $0.96927(19)$ & $0.0446(8)$ \\
\hline C18 & $0.2862(2)$ & $-0.0717(3)$ & $0.9976(2)$ & $0.0483(9)$ \\
\hline H18 & 0.329241 & -0.093723 & 0.977246 & $0.058^{*}$ \\
\hline C19 & 0.2781 & $-0.1406(3)$ & $1.05700(19)$ & $0.0454(8)$ \\
\hline $\mathrm{C} 20$ & $0.2178(2)$ & $-0.1105(3)$ & $1.08900(19)$ & $0.0455(8)$ \\
\hline $\mathrm{H} 20$ & 0.214185 & -0.157660 & 1.129021 & $0.055^{\star}$ \\
\hline $\mathrm{C} 21$ & $0.0999(2)$ & $0.0252(3)$ & $1.09780(18)$ & $0.0442(8)$ \\
\hline $\mathrm{H} 2$ & 0.100945 & -0.024969 & 1.138307 & $0.053^{*}$ \\
\hline $\mathrm{C} 22$ & $0.2917(3)$ & $0.0701(4)$ & $0.8731(2)$ & $.0736(13)$ \\
\hline $\mathrm{H} 22 \mathrm{~A}$ & 0.356165 & 0.073614 & 0.911092 & $0.110^{*}$ \\
\hline $\mathrm{H} 22 \mathrm{~B}$ & 0.282484 & 0.126302 & 0.831147 & $0.110^{*}$ \\
\hline $\mathrm{H} 22 \mathrm{C}$ & 0.277343 & -0.010774 & 0.852189 & $0.110^{*}$ \\
\hline
\end{tabular}

Table 2 (continued)

\begin{tabular}{|c|c|c|c|c|}
\hline Atom & $x$ & $y$ & $z$ & $U_{\text {iso }}{ }^{*} / U_{\text {eq }}$ \\
\hline $\mathrm{C} 23$ & $0.0547(3)$ & $0.1919(4)$ & $0.6815(3)$ & $0.0648(11)$ \\
\hline $\mathrm{H} 23$ & 0.118089 & 0.206822 & 0.694459 & $0.078^{*}$ \\
\hline $\mathrm{C} 24^{\mathrm{a}}$ & $-0.0876(10)$ & $0.123(2)$ & $0.5919(11)$ & $0.148(11)$ \\
\hline $\mathrm{H} 24 \mathrm{~A}^{\mathrm{a}}$ & -0.088489 & 0.046218 & 0.566743 & $0.222^{*}$ \\
\hline $\mathrm{H} 24 \mathrm{~B}^{\mathrm{a}}$ & -0.139629 & 0.171695 & 0.558037 & $0.222^{\star}$ \\
\hline $\mathrm{H} 24 \mathrm{C}^{\mathrm{a}}$ & -0.092693 & 0.108735 & 0.640724 & $0.222^{\star}$ \\
\hline $\mathrm{C} 25$ & $0.0348(4)$ & $0.2003(5)$ & $0.5464(3)$ & $0.1039(18)$ \\
\hline $\mathrm{H} 25 \mathrm{~A}$ & 0.009378 & 0.273696 & 0.517460 & $0.156^{\star}$ \\
\hline $\mathrm{H} 25 \mathrm{~B}$ & 0.015678 & 0.132048 & 0.511217 & $0.156^{\star}$ \\
\hline $\mathrm{H} 25 \mathrm{C}$ & 0.102366 & 0.205234 & 0.570549 & $0.156^{*}$ \\
\hline$C 24 A^{a}$ & $-0.1020(8)$ & $0.195(3)$ & $0.5768(12)$ & $0.142(11)$ \\
\hline$H 24 D^{a}$ & -0.130286 & 0.121621 & 0.548696 & $0.212^{*}$ \\
\hline $\mathrm{H} 24 \mathrm{E}^{\mathrm{a}}$ & -0.123318 & 0.263070 & 0.541864 & $0.212^{\star}$ \\
\hline $\mathrm{H} 24 \mathrm{~F}^{\mathrm{a}}$ & -0.119808 & 0.205367 & 0.619862 & $0.212^{\star}$ \\
\hline
\end{tabular}

accupancy: 0.5 .

their interesting magnetic properties [7-9]. For example new sandwich-type heterometallic $\mathrm{Zn}^{\mathrm{II}}-\mathrm{Ln}^{\mathrm{III}}$ clusters based on a methoxy substituted salicylamide salen-like ligand $\left[\mathrm{Zn}_{2} \mathrm{Er}_{3}(\mathrm{HL})_{2}\left(\mathrm{H}_{2} \mathrm{~L}\right)_{2}(\mathrm{OH})_{2}\left(\mathrm{NO}_{3}\right)_{3}\right]\left(\mathrm{NO}_{3}\right)_{2.2} \mathrm{CH}_{3} \mathrm{OH}\left(\mathrm{Zn}_{2} \mathrm{Er}_{3}\right),\left[\mathrm{Zn}_{2}\right.$ $\left.\mathrm{Yb}_{3}(\mathrm{HL})_{2}\left(\mathrm{H}_{2} \mathrm{~L}\right)_{2}(\mathrm{OH})_{2}\left(\mathrm{NO}_{3}\right)_{3}\right]\left(\mathrm{NO}_{3}\right)_{2.2} \mathrm{CH}_{3} \mathrm{OH}\left(\mathrm{Zn}_{2} \mathrm{Yb}_{3}\right), \mathrm{H}_{3} \mathrm{~L}=$ 1-(2-hydroxy-4-methoxy-benzamido)-2-(2-hydroxy-3-methoxybenzylideneamino)-ethane were reported. The magnetic susceptibility measurements shows that $\mathrm{Zn}_{2} \mathrm{Er}_{3}$ displays antiferromagnetic interactions with zero field slow magnetization relaxation diagnostic of single-molecule-magnet [10]. The Ghosh group reported three trinuclear hetero-metallic complexes with a reduced salen type Schiff base ligand and further investigated their maganetic properties [11].

Herein, we report the synthesis and crystal structure of a heterometallic NiNa complex, which can maybe act as a suitable assemble segment to prepare multimetallic moleculebased magnetic material.

The Ni ion is four-coordinated by a $\mathrm{N}_{2} \mathrm{O}_{2}$ unit from the Schiff-base ligand, forming a square planar geometry. The $\mathrm{Ni}-\mathrm{O}$ and $\mathrm{Ni}-\mathrm{N}$ bond lengths are almost equal to each other with the average value of $1.841 \AA$ and $1.852 \AA$, respectively. The mean deviation of the plane formed by NiN2O2 is only 0.01(2) $\AA$, indicating that these five atoms are in a perfect plane. The coordination sphere of the $\mathrm{Na}$ ion is a distorted pentagonal bipyramid, in which the five equatorial positions are from a $\mathrm{O}_{4}$ unit of the Schiff-base ligand and one $\mathrm{O}$ atom of $\mathrm{ClO}_{4}{ }^{-}$ion and the axial ones occupied by one $\mathrm{O}$ atom of the coordinated DMF molecule and one $\mathrm{O}$ atom of $\mathrm{ClO}_{4}{ }^{-}$ion. The distances between the $\mathrm{Na}$ atom and the seven $\mathrm{O}$ atoms are with slight difference within a narrow range from 2.287(4) to 2.627(4) A. Additionally, this complex forms a supramolecular three-dimensional structure based on weak intermolecular $\mathrm{C}-\mathrm{H} \cdots \mathrm{O}$ and $\mathrm{C}-\mathrm{H} \cdots \mathrm{Cl}$ interactions. 
Acknowledgements: We gratefully thank the financial support of the Shandong Provincial Natural Science Foundation (ZR2015BM005), Science and Technology Program of Weifang (2017GX014).

\section{References}

1. Bruker. APEX2, SAINT and SADABS. Bruker AXS Inc., Madison, WI, USA (2012).

2. Sheldrick, G. M.: A short history of SHELX. Acta Crystallogr. A64 (2008) 112-122.

3. Dolomanov, O. V.; Bourhis, L. J.; Gildea, R. J.; Howard, J. A. K.; Puschmann, H.: OLEX2: a complete structure solution, refinement and analysis program. J. Appl. Crystallogr. 42 (2009) 339-341.

4. Dasa, P.; Linertb, W.: Schiff base-derived homogeneous and heterogeneous palladium catalysts for the Suzuki-Miyaura reaction. Coord. Chem. Rev. 311 (2016) 1-23.

5. Khan, M. I.; Khan, A.; Hussain, I.; Khan, M.; Gul, S.; Iqbal, M.; Rahman, I.; Khuda, F.: Spectral, XRD, SEM and biological properties of new mononuclear Schiff base transition metal complexes. Inorg. Chem. Commun. 35 (2013) 104-109.

6. Zhu, W.; Zhang, S.; Cui, C.; Bi, F.; Ke, H.; Xie, G.; Chen, S.: New dinuclear cobalt (II, III) and tetranuclear manganese (III) complexes assembled by a polydentate Schiff-base ligand: synthesis, structure and magnetic properties. Inorg. Chem. Commun. 46 (2014) 315-319.

7. Miyasaka, H.; Saitoh, A.; Abe, S.: Magnetic assemblies based on Mn(III) salen analogues. Coord. Chem. Rev. 251 (2007) 2622-2664.

8. Andruh, M.: Compartmental Schiff-base ligands-a rich library of tectons in designing magnetic and luminescent materials. Chem. Commun. 47 (2011) 3025-3042.

9. Biswas, A.; Ghosh, M.; Lemoine, P.; Sarkar, S.; Hazra, S.; Mohanta, S.: Syntheses, structures and magnetic properties of trinuclear $\mathrm{Cu}^{\prime \prime} \mathrm{M}^{\prime \prime} \mathrm{Cu}$ " $(\mathrm{M}=\mathrm{Cu}, \mathrm{Ni}, \mathrm{Co}$ and $\mathrm{Fe})$ and tetranuclear [2*1+1*2] $\mathrm{Cu}^{\prime \prime} \mathrm{Mn}^{\mathrm{N}}-2 \mathrm{Cu}$ " complexes derived from a compartmental ligand: the schiff base 3-methoxysalicylaldehyde diamine can also stabilize a cocrystal. Eur. J. Inorg. Chem. 20 (2010) 3125-3134.

10. Liu, P.; Wang, C.; Zhang, M.; Song, X.: Pentanuclear sandwich-

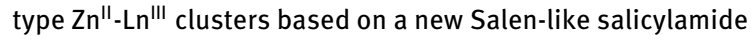
ligand: structure, near-infrared emission and magnetic properties. Polyhedron 129 (2017) 133-140.

11. Hazari, A.; Gmeiz-Garcia, C.; Drew, M.; Ghosh, A.: Rare examples of diphenoxido-bridged trinuclear $\mathrm{Ni}^{\mathrm{il}} 2 \mathrm{Fe} \mathrm{III}^{\mathrm{II}} \mathrm{com}$ plexes with a reduced salen type Schiff base ligand: Structures and magnetic properties. Polyhedron 138 (2017) 145-153. 